\title{
Development of a Genomics-Based LAMP (Loop-Mediated Isothermal Amplification) Assay for Detection of Pseudomonas fuscovaginae from Rice
}

Gavin J. Ash, Graham Centre for Agricultural Innovation (an alliance between Charles Sturt University and NSW DPI), School of Agricultural and Wine Sciences, Charles Sturt University, Wagga Wagga 2678, NSW, Australia; Jillian M. Lang and Lindsay R. Triplett, Colorado State University, Department of Bioagricultural Sciences \& Pest Management, Fort Collins 80523-1177; Benjamin J. Stodart, Graham Centre for Agricultural Innovation; Valérie Verdier, Institut de Recherche pour le Développement, UMR Résistance des Plantes aux Bioagresseurs, IRD-CIRAD-UM2, 34394 Montpellier Cedex 5, France; Casiana Vera Cruz, International Rice Research Institute, DAPO Box 7777, Metro Manila, Philippines; Philippe Rott, CIRAD, UMR BGPI, F-34398 Montpellier, France; and Jan E. Leach, Colorado State University, Department of Bioagricultural Science \& Pest Management

\begin{abstract}
Ash, G. J., Lang, J. M., Triplett, L. R., Stodart, B. J., Verdier, V., Vera Cruz, C., Rott, P., and Leach, J. E. 2014. Development of a genomics-based LAMP (loop-mediated isothermal amplification) assay for detection of Pseudomonas fuscovaginae from rice. Plant Dis. 98:909-915.

The vast amount of data available through next-generation sequencing technology is facilitating the design of diagnostic marker systems. This study reports the use of draft genome sequences from the bacterial plant pathogen Pseudomonas fuscovaginae, the cause of sheath brown rot of rice, to describe the genetic diversity within a worldwide collection of strains representing the species. Based on a comparative analysis with the draft sequences, primers for a loop-mediated isothermal amplification (LAMP) assay were developed to identify P. fuscovagi-

nae. The assay reported here reliably differentiated strains of $P$. fuscovaginae isolated from rice from a range of other bacteria that are commonly isolated from rice and other plants using a primer combination designated Pf8. The LAMP assay identified $P$. fuscovaginae purified DNA, live or heat-killed cells from pure cultures, and detected the bacterium in extracts or exudates from infected host plant material. The $P$. fuscovaginae LAMP assay is a suitable diagnostic tool for the glasshouse and laboratory and could be further developed for in-field surveys.
\end{abstract}

Pseudomonas is a large and diverse genus in the $\gamma$ subclass of Proteobacteria (26). The genus contains plant pathogens, opportunistic human pathogens, plant commensals, biological control agents, and environmental microbes $(3,17,37)$. The diversity of this group is reflected in the large pan-genome and relatively small core genome (26). Pseudomonas fuscovaginae, Miyajima, Tanii and Akita nom. rev., the cause of sheath brown rot disease of rice (27), belongs to the $P$. fluorescens group and the subgroup $P$. asplenii, based on partial sequences of four housekeeping genes (29). Sheath brown rot of rice was first reported in Hokkaido, Japan in 1982 (41) and has since been reported from most rice-growing regions of the world, including the Philippines (11), Mexico (13), Nepal (36), Madagascar (34), Latin America (47), and Australia (10). In addition to sheath browning, $P$. fuscovaginae can cause a systemic discoloration of sheath in seedlings, grain discoloration and panicle sterility in adult plants (47), and total yield loss with very severe infection (34). In addition to rice, $P$. fuscovaginae infects maize, sorghum, and wheat $(13,14)$.

A large number of other bacterial species are associated with grain and glume discoloration in rice as well as sheath browning $(2,9,19)$, and these symptoms are often associated with mixed microbial populations in the field. Symptoms of sheath brown rot caused by $P$. fuscovaginae can be confused not only with symptoms caused by other common bacterial pathogens of rice, including Burkholderia glumae, B. cepacia, Pantoea ananatis, and Acidovorax avenae, but also with some fungal pathogens of rice such as Sarocladium sp. and Dreschlera sp. (48). Accurate identification of causal organisms of diseases is fundamental to declaration of pest-free areas and for identification of resistant germplasm (1). Therefore, there is a need for a rapid, robust

Corresponding author: G. J. Ash, E-mail: gash@csu.edu.au

Accepted for publication 13 December 2013.

http://dx.doi.org/10.1094/PDIS-09-13-0957-RE

(C) 2014 The American Phytopathological Society diagnostic assay for Pseudomonas fuscovaginae that could be deployed in the field.

Definitive diagnosis of the casual agent of sheath brown rot traditionally involves a polyphasic approach that includes pathogen isolation, morphological studies, and biochemical tests such as BACTID (5) and DNA sequencing (2,10). Recently, loop-mediated isothermal amplification (LAMP), a rapid and sensitive technique first developed by Notomi et al. (31), has been adopted widely as a diagnostic tool for human clinical samples (28). The amplification of DNA using this technique combines a Bst DNA polymerase with strand-displacement activity, four to six flanking primers, and amplification temperatures between 60 and $65^{\circ} \mathrm{C}$ (31). The technique is less sensitive to inhibitors than polymerase chain reaction (PCR) and, hence, is applicable for a wide variety of clinical and field samples (21). Recently, the technique has been used for the detection of human, animal, and plant pathogens $(15,16,24,30,44$ $46)$. With no requirement for temperature cycling or secondary steps for visualizing results, the technique can be used in the field with minimal training, making LAMP more feasible for use in developing countries than conventional PCR (16). Diagnosis can be rapidly accomplished within 15 to $60 \mathrm{~min}$. The availability of whole-genome sequences for many species of plant pathogens allows the rapid development of primers for assays such as LAMP, using coding sequences that are conserved within the target species. This comparative genomic approach, using draft genomes, to find selective targets for LAMP was recently used to develop a selective assay for Erwinia amylovora (7).

Here, we describe the use of draft genome sequences for $P$. fuscovaginae to assess genomic diversity of representative strains and guide the development of a selective and sensitive LAMP detection assay that is applicable for a global collection of $P$. fuscovaginae.

\section{Materials and Methods}

Whole-genome sequences of $\boldsymbol{P}$. fuscovaginae. Draft wholegenomic sequences of $P$. fuscovaginae used included UPB0736 (accession number AIEU00000000) (32), CB98818 (accession number ALAQ01000000) (42), and DAR 77795 and DAR 77800 (Bioproject numbers PRJDB1417 and PRJDB 1418, respectively). 
A full genomic sequence of a closely related Pseudomonad isolated from rice in Cambodia (DAR 77817) (9) was included as a comparison (Bioproject number PRDJB1419). Additionally, a draft genome sequence of a strain of $P$. fuscovaginae collected in the Philippines in 2000 (SE1; 12) was produced on an Illumina GAII sequencer at the University of California, Davis. Short-read trimming, filtering for quality, and shuffling were performed using Perl scripts from the FastX toolkit (version 0.0.13; http://hannonlab. cshl.edu/fastx_toolkit/). Reads with a quality score of less than 20 over $80 \%$ of the read were removed, and reads were trimmed to 55 bp. Reads were assembled de-novo using Velvet 1.1.06 (Zerbino and Birney 2008) with parameters optimized by VelvetOptimiser.pl, using a hash length of 45 and a k-mer length of 61 .

Genomic diversity in $P$. fuscovaginae. 16s ribosomal RNA sequence comparison. Sequences (1,394 bp) of the $16 \mathrm{~s}$ ribosomal RNA (rRNA) gene from 22 Pseudomonas strains were generated and aligned using the ClustalW algorithm within MEGA 5.2 (40). The evolutionary history was inferred by using the maximum-likelihood method based on the Tamura-Nei model (39). Initial trees for the heuristic search were obtained by applying the neighborjoining method to a matrix of pairwise distances estimated using the maximum composite likelihood approach. All positions containing gaps and missing data were eliminated. In total, 1,313 positions were present in the final dataset. Evolutionary analyses were conducted in MEGA5 (5.2).

Multilocus sequence typing. The sequence of seven housekeeping genes ( $\operatorname{acs} A$ [acetyl coenzyme A synthetase], aroE [shikimate dehydrogenase], guaA [GMP synthase], mutL [DNA mismatch repair protein], nuoD [NADH dehydrogenase I chain $\mathrm{C}, \mathrm{D}]$, ppsA [phosphoenolpyruvate synthase], and $\operatorname{trpE}$ [anthralite synthetase component I]) from draft sequences of $P$. fuscovaginae strains and a Pseudomonas sp. from Cambodia were compared with the reference genome of $P$. fluorescens using multilocus sequence typing (MLST) 1.5 (http://cge.cbs.dtu.dk/services/MLST/) (23); additionally, the sequences of $r p o B$ for each of the strains were used. The concatenated sequences were aligned using MEGA 5.2 (40) and the evolutionary history was inferred by using the maximum-likelihood method based on the JTT matrix-based model (20). Sequences for other Pseudomonas spp. (P. syringae pv. tomato DC3000 [ICMP 3443], $P$. aeruginosa DK2, P. stutzeri [ATCC 17588], $P$. fluorescens A506, $P$. putida BIRD1, $P$. fragi A22, and $P$. chlororaphis 06) were included for comparison.

Whole-genome comparison. Concatenated whole genomes of $P$. fuscovaginae and other Pseudomonas spp. (Pseudomonas sp. [DAR 77817], $P$. syringae pv. tomato DC3000 [ICMP 3443], $P$. aeruginosa DK2, P. stutzeri [ATCC 17588], P. fluorescens A506, and $P$. putida BIRD1) were compared and a phylogenetic tree was constructed using the programs EDGAR (6) and DARwin (33).

Plant growth and inoculation conditions. Seed of rice (Oryza sativa 'M202') were germinated overnight before being placed in a compost mix (pH 4 to 4.5 ) in $100-\mathrm{cm}^{2}$ pots in a controlled-environment glasshouse (65 to $70 \%$ relative humidity; 26 and $21{ }^{\circ} \mathrm{C}$ day and night, respectively; and light at 400 to $600 \mathrm{~W} / \mathrm{m}^{2}$ for a maximum of $12 \mathrm{~h}$ ). The pots were fertilized with a mixture of Algospeed (COMPO EXPERT; 176 g/liter) and Ferveg E13 (Angibaud-France; $12.5 \mathrm{~g} /$ liter). This stock solution was diluted (1\%) and 1 liter of the diluted solution was applied once a week to 200 plants. The flag leaf sheath of 10 rice plants (growth stage 51, beginning of panicle emergence) was inoculated with $P$. fuscovaginae
(DAR 77795) using a needle dipped in a cell suspension (approximately $1 \times 10^{6}$ cells $/ \mathrm{ml}$ ), as described by Adorada et al. (1). The plants were then misted for $24 \mathrm{~h}$ at $23^{\circ} \mathrm{C}$ in the dark before being returned to the glasshouse for symptom development. Five control plants were wounded with a needle dipped in sterile distilled water.

LAMP. Primer design. A MUMmer alignment strategy (22) was used to identify four conserved sequences (Table 1) shared between two disparate strains of $P$. fuscovaginae (SE1 from the Philippines and ICMP 9996 from Madagascar) but absent in the genome sequences of $P$. syringae B728A, $P$. fulva strain $12-\mathrm{X}, B$. glumae BGR1, A. citrulli AAC001, Xanthomonas oryzae pv. oryzae PXO99A, and X. campestris strain 8004 (accessions NC_007085, NC_015556, NC_012724 and NC_012721, NC_008752, NC_010717, and NC_007086, respectively). The corresponding regions were then extracted from the sequences of the above two strains of $P$. fuscovaginae plus an Australian strain (DAR 77795), the type strain from Japan (ICMP 5940), and a Chinese strain (CB 98818). These sequences were aligned with MEGA 5.2 (40) and the consensus sequence was used to design primers using LAMP Designer 1.02 (LAMP Designer; Premier Biosoft International; Table 2). These consisted of the forward outer (F3), reverse outer (B3), the forward and reverse inner primers (FIP and FIB, respectively) and the forward and reverse loop primers (LoopF and LoopB, respectively). Primers were synthesized by Integrated DNA Technologies. Initially, the F3 and B3 primers of each set were checked for amplification of DNA of strains DAR 77795, ICMP 9996, and SE1 by conventional PCR using $P$. syringae DNA as a negative control. Reactions $(25 \mu \mathrm{l})$ consisted of $3 \mathrm{mM} \mathrm{MgCl} 2,0.5 \mathrm{mM}$ each dNTP, $4 \mu \mathrm{M}$ F3 primer, 4 $\mu \mathrm{M}$ B3 primer, and 1 unit of Taq polymerase (New England BioLabs) in a $1 \times$ standard buffer (New England Biolabs). The temperature profile used was $3 \mathrm{~min}$ at $94^{\circ} \mathrm{C}$; followed by 34 cycles of $30 \mathrm{~s}$ at $94^{\circ} \mathrm{C}, 30 \mathrm{~s}$ at $50^{\circ} \mathrm{C}$, and $90 \mathrm{~s}$ at $72^{\circ} \mathrm{C}$; and a final elongation cycle at $72^{\circ} \mathrm{C}$ for $3 \mathrm{~min}$. PCR product $(7 \mu \mathrm{l})$ was mixed with $3 \mu \mathrm{l}$ of $5 \times$ loading dye and loaded on a $1 \%$ agarose gel containing $1 \times$ Gel Red (Biotium, Inc.). The gels were run at $85 \mathrm{~V}$ for $45 \mathrm{~min}$ and amplicons were visualized using a Syngene transilluminator (Synoptics Ltd.).

After confirmation of DNA amplification with F3/B3, the full set of LAMP primers was evaluated. These included LAMP primer sets Pf3, Pf4, Pf7, Pf8, and Pf9, which were used for amplification of all samples shown in Table 3 . Reactions $(12 \mu \mathrm{l})$ were composed of $10 \mathrm{ng}$ of template DNA, 7.2 $\mu$ of Isothermal Master Mix (Optigene Limited), $3.2 \mathrm{nM}$ each F3/B3 primer, $0.32 \mu \mathrm{M}$ each FIP/BIP primer, $1.6 \mu \mathrm{M}$ each LoopF/LoopB primer, and $1.8 \mu \mathrm{l}$ of water. All amplifications were performed in a Genie II (OptiGene Limited). The temperature profile was $65^{\circ} \mathrm{C}$ for $30 \mathrm{~min}$ followed by $85^{\circ} \mathrm{C}$ for 5 min to inactivate the enzyme. A reaction with no template DNA and one with DNA of $P$. syringae $(10 \mathrm{ng} / \mu \mathrm{l})$ were used as negative controls.

Sensitivity analysis. To determine the sensitivity of the LAMP assay, a 10 -fold serial dilution $\left(1 \times 10^{0}\right.$ to $\left.1 \times 10^{-6}\right)$ of $P$. fuscovaginae SE1 DNA $(10 \mathrm{ng} / \mu \mathrm{l})$ was prepared. These dilutions (along with a no-DNA template control) were amplified in a real-time PCR machine (CFX Connect; Bio-Rad) under the following conditions: $1 \mathrm{ng}$ of template DNA, $32 \mathrm{nM}$ each F3/B3 primer, $0.32 \mu \mathrm{M}$ each FIP/BIP primer, $1.6 \mu \mathrm{M}$ each LoopF/LoopB primer, and $7.2 \mu \mathrm{l}$ of Isothermal Master Mix (Optigene Limited) up to a total volume of $12 \mu \mathrm{l}$. The cycle consisted of 64 to $65^{\circ} \mathrm{C}$ for $1 \mathrm{~h}$ and a melt curve

Table 1. Pseudomonas fuscovaginae genes with conserved sequences in other Pseudomonas spp. identified through the genomic comparison of strains SE 1 and ICMP 9996 using DNA sequence analysis

\begin{tabular}{|c|c|c|c|c|c|}
\hline $\begin{array}{l}\text { Sequence } \\
\text { name }\end{array}$ & $\begin{array}{c}\text { Length } \\
\text { (bp) }\end{array}$ & Function & Isolate & $\begin{array}{c}\text { Accession } \\
\text { number }\end{array}$ & $\begin{array}{l}\text { Nucleic acid } \\
\text { identity }(\%)\end{array}$ \\
\hline SEQ001 & 3246 & Flagellar rod assembly protein/muramidase FlgJ & P. protegens $\mathrm{Pf}-5$ chromosome, complete genome & NC_004129 & 77 \\
\hline SEQ371 & 396 & Chromosomal replication initiator protein & P. putida UW4 chromosome, complete genome & NC_019670.1 & 83 \\
\hline SEQ483 & 531 & Hypothetical protein & $\begin{array}{l}\text { P. brassicacearum subsp. brassicacearum NFM421 } \\
\text { chromosome, complete genome }\end{array}$ & NC_015379.1 & 88 \\
\hline SEQ992 & 420 & Chromosomal replication initiator protein & P. putida UW4 chromosome, complete genome & NC_019670.1 & 85 \\
\hline
\end{tabular}


cycle of 65 to $95^{\circ} \mathrm{C}$ (increments of $0.5^{\circ} \mathrm{C}$ per $5 \mathrm{~s}$ ). All reactions were performed twice. A sensitivity assay was also performed on live cells. A 10-fold dilution of a suspension of $P$. fuscovaginae (DAR 77795) grown on King's B agar (KBA) was made in sterile distilled water. The serially diluted cells were used in a LAMP assay as described above, except the assay was performed in a StrataGene Real-Time PCR machine (MX3005) at $60^{\circ} \mathrm{C}$. Each of the dilutions $(100 \mu \mathrm{l})$ was spread on KBA and incubated for $48 \mathrm{~h}$ at $28^{\circ} \mathrm{C}$ to determine the concentration of cells in the suspension. The serial dilutions were replicated four times and the experiment was conducted twice.

Specificity analysis. To determine the specificity of the LAMP primers (Pf8), three pools of DNA from bacteria (10 per pool) isolated from rice seed were made (Table 4). LAMP reactions were run on these pools with and without DNA of $P$. fuscovaginae SE1 using the real-time LAMP parameters described above. All reactions were performed twice. Additionally, DNA from Pseudomonads isolated from rice in Cambodia were used individually in LAMP reactions (Table 4).
LAMP analysis of infected plants. A $5-\mathrm{mm}^{2}$ section of leaf sheath was excised from both inoculated and water control plants 5 days after inoculation (five replicate plants). The plant material was submerged in $200 \mu \mathrm{l}$ of sterile distilled water in an Eppendorf tube and crushed using a sterile micropestle (Eppendorf International). A 10-fold serial dilution of the macerated tissue was made in sterile distilled water. Sample $(1 \mu \mathrm{l})$ from each of the $10^{-1}, 10^{-2}$, and $10^{-3}$ dilutions was used for the LAMP assay, as described above. A no-template control and an assay containing $10 \mathrm{ng}$ of purified DNA of strain DAR 77795 were used as negative and positive controls, respectively. The assay was performed in a StrataGene Real-Time PCR machine (MX3005), as described above. All reactions were performed twice.

\section{Results}

Whole-genome sequence of $P$. fuscovaginae strain SE1. The genome statistics for $P$. fuscovaginae strain SE1 are shown in Table 5. This whole-genome shotgun project was deposited at DDBJ/EMBL/GenBank under the accession AQOH00000000.

Table 2. Loop-mediated isothermal amplification primers developed for the detection of Pseudomonas fuscovaginae ${ }^{\mathrm{a}}$

\begin{tabular}{|c|c|c|c|}
\hline Primer set & Single primer & Sequence $\left(5^{\prime}-3^{\prime}\right)$ & Relative position \\
\hline \multirow[t]{6}{*}{ Pf3 } & F3 & GAATTCCGTTCCTATGATTCCT & 1,080 \\
\hline & B3 & TTGAGCAGACTCATGATTCAG & 1,346 \\
\hline & FIP & TCACGCACAAACTGTTCTGGTTGCAGAGCAATAATCGCTAT & $\ldots$ \\
\hline & BIP & AAAGGCCGGGTACGCAACTCTGGTAGCTCTTCATCTGT & $\ldots$ \\
\hline & LoopF & GCCGACTTCAGCACATCT & 1,174 \\
\hline & LoopB & TACGCCAGCAAGATCACG & 1,236 \\
\hline \multirow[t]{6}{*}{ Pf4 } & F3 & CAGGACGCCATCAAGTAC & 2,778 \\
\hline & B3 & CCGGTCATCGAAATGGTATAG & 3,076 \\
\hline & FIP & AGGTTCAGGGTATTGTTCTGGCGAGCTACACACTGACCGA & $\ldots$ \\
\hline & BIP & TGGTCGACTCGTCGGGTAATGGTCTGTACCGTAAAGGT & $\ldots$ \\
\hline & LoopF & GCCCATGAGATTGCCCTT & 2,894 \\
\hline & LoopB & GTGATGGACAACACCGTCA & 2,970 \\
\hline \multirow[t]{6}{*}{ Pf7 } & F3 & CATGGGTTGCTCCTTTGAT & 69 \\
\hline & B3 & CACATTGCTGTGAGCCTT & 302 \\
\hline & FIP & ATTGTGCGCGTGTAGGCATAGCGTATCCTGTGCATTGT & $\ldots$ \\
\hline & BIP & CAATCCCGGAACGCTGGTTAAAGATGGCATCGAACAGC & 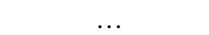 \\
\hline & LoopF & AACGGTGTTTGCTGGTAGT & 175 \\
\hline & LoopB & CGTCAGGCAACGTCTGAT & 239 \\
\hline \multirow[t]{6}{*}{ Pf8 } & F3 & TGTGTTCAGTTCCTTCATCG & 197 \\
\hline & B3 & TAACAGCGTCACGTTCTTC & 473 \\
\hline & FIP & GATCATCAGCGGGACTGTCAGGGTGAGGTCTACGAGAAATTC & $\ldots$ \\
\hline & BIP & CGACGGCGATTTCGTTGCGTTATACAACAACGCTTGCC & \\
\hline & LoopF & TGAGAAACGCCTTCTGGTC & 306 \\
\hline & LoopB & TGTGCTGTGCCTGATTCTT & 365 \\
\hline \multirow[t]{6}{*}{ Pf9 } & F3 & TCGTCGAGGTCGATATGG & 138 \\
\hline & B3 & GCGAAGAGGAAGAACTCAG & 372 \\
\hline & FIP & CCTCGGAACGCTGAAGAGCTTACGCATGTATTGCTGACT & $\ldots$ \\
\hline & BIP & CGAGCACCTGTTGGTGGATTGTTCAACGACTTCCTGG & $\ldots$ \\
\hline & LoopF & CACCAGCCGATGGTAGAC & 196 \\
\hline & LoopB & TCGCCGGGATATTCCATTTC & 280 \\
\hline
\end{tabular}

${ }^{a}$ Primer sets Pf3 and Pf4 were based on sequence SEQ001, Pf7 was based on SEQ371, Pf8 was based on SEQ483, and Pf9 was based on SEQ992.

Table 3. Isolates of Pseudomonas fuscovaginae, Pseudomonas sp., and P. syringae used in the initial specificity testing of loop-mediated isothermal amplification (LAMP) primers ${ }^{\mathrm{a}}$

\begin{tabular}{|c|c|c|c|c|c|c|c|c|c|}
\hline \multirow[b]{2}{*}{ Accession number } & \multirow[b]{2}{*}{ Species } & \multirow[b]{2}{*}{ Origin } & \multirow[b]{2}{*}{ Host } & \multirow[b]{2}{*}{ Notes } & \multicolumn{5}{|c|}{ LAMP primer set } \\
\hline & & & & & Pf3 & Pf4 & Pf7 & Pf8 & Pf9 \\
\hline DAR 77795 & P. fuscovaginae & Australia & Oryza sativa & DNA & + & + & - & + & + \\
\hline DAR 77797 & P. fuscovaginae & Australia & O. sativa & DNA & + & + & nt & + & - \\
\hline DAR 77817 & Pseudomonas sp. & Cambodia & O. sativa & DNA & - & - & $\mathrm{nt}$ & - & - \\
\hline DAR 77794 & Pseudomonas sp. & Cambodia & O. sativa & DNA & - & - & nt & - & - \\
\hline UPB264b & P. fuscovaginae & Burundi & O. sativa & Heat-killed cells & + & + & nt & + & - \\
\hline ICMP 5940 & P. fuscovaginae & Japan & O. sativa & Heat-killed cells & + & + & nt & + & - \\
\hline UPB407 & P. fuscovaginae & Burundi & Leersia hexandra & Heat-killed cells & + & - & nt & - & - \\
\hline UPB0735 & P. fuscovaginae & Burundi & O. sativa & Heat-killed cells & + & + & nt & + & - \\
\hline ICMP 9996 & P. fuscovaginae & Madagascar & O. sativa & Heat-killed cells & + & \pm & nt & + & - \\
\hline UPB898 & P. fuscovaginae & Colombia & O. sativa & Heat-killed cells & - & - & nt & + & - \\
\hline SE1 & P. fuscovaginae & Philippines & O. sativa & Heat-killed cells & + & - & - & + & + \\
\hline$N C P P B 1242$ & P. syringae pv. syringae & Australia & Panicum milaceum & DNA & - & - & - & - & - \\
\hline
\end{tabular}

${ }^{\text {a }}$ All assays were conducted at least twice; $\mathrm{nt}=$ not tested. 
The version described in this article is the first version, AQOH01000000.

Genomic diversity of $\boldsymbol{P}$. fuscovaginae. The $P$. fuscovaginae strains clustered with isolate strain of $P$. asplenii (with a bootstrap value of $96 \%$ ) and are clearly different from the representative sequences from the other species of Pseudomonas, based on the phylogeny generated from the 16s rRNA gene sequences (Fig. 1). The Pseudomonas sp. (ICMP $17674=$ DAR 77817) isolated from rice in Cambodia (9) was more closely related to $P$. putida. $P$. fuscovaginae strains ICMP 9996, CB98818, DAR 77795, ICMP 5940, and DAR 77800 clustered closely within two subclades of
Table 5. Draft genome assembly statistics for Pseudomonas fuscovaginae strain SE 1

\begin{tabular}{lc}
\hline Total PF reads & $24,345,762$ \\
Total Mbp reads used & 1,232 \\
Sequence coverage & $188 \times$ \\
N50 & 92,359 \\
Number of contigs & 692 \\
Maximum contig length (bp) & 305,205 \\
Total contig length (bp) & $6,558,325$ \\
\hline
\end{tabular}

Table 4. Bacterial strains used in this study to establish loop-mediated isothermal amplification (LAMP) assay sensitivity ${ }^{\mathrm{a}}$

\begin{tabular}{|c|c|c|c|c|}
\hline Strain & Country of origin & Host & Species & Notes, source \\
\hline NCPPB3112 & Brazil & Canna indica & Acidovorax avenae pv. avenae & $\mathrm{NCPPB}^{\mathrm{b}}$ \\
\hline $94-21$ & United States & Citrullus lanatus & A. avenae pv. citrulli & R. Walcott \\
\hline 3549 & United States & Saccharum officinarum & Burkolderia andropogonis & L. E. Claflin \\
\hline $\mathrm{O} 187$ & United States & Allium сера & B. gladioli & H. F. Schwartz \\
\hline B473 & United States & Phaseolous sp. & Curtobacterium flaccumfaciens & H. F. Schwartz \\
\hline O121 & United States & A. сера & Enterobacter sp. & H. F. Schwartz \\
\hline $\mathrm{O} 174$ & United States & A. сера & Enterobacter $\mathrm{sp}$. & H. F. Schwartz \\
\hline 112 & United Kingdom & Pyrus communis & Erwinia herbicola & L. E. Claflin \\
\hline $\mathrm{O} 158$ & United States & A. сера & Pantoea ananatis & H. F. Schwartz \\
\hline ATCC10844 & United States & Lactuca sativa & Pseudomonas marginalis & H. F. Schwartz \\
\hline NCPPB 1242 & United States & Panicum milaceum & Pseudomonas syringae pv. syringae & L. E. Claflin \\
\hline M108 & United States & Solanum lycopersicum & Pseudomonas syringae pv. syringae & H. F. Schwartz \\
\hline M72 & United States & Capsicum аппиит & P. syringae pv. syringae & H. F. Schwartz \\
\hline ATCC13223 & United States & Phaseolus coccineus & Pseudomonas viridiflava & H. F. Schwartz \\
\hline K60 & United States & S. lycopersicum & Ralstonia solanacearum & J. E. Leach \\
\hline NCPPB 2417 (4429) & New Zealand & Sorghum bicolor & Xanthomonas axonopodis pv. holcicola & L.E. Claflin \\
\hline 2919 & Réunion Island & Mangifera sp. & X. axonopodis pv. mangiferaeindicae & L. Gagnevin \\
\hline $\mathrm{X}-1$ & United States & Pelargonium sp. & X. campestris pv. pelargonii & J. E. Leach \\
\hline $85-10$ & United States & Capsicum sp. & X. campestris pv. vesicatoria & A. J. Bogdanove \\
\hline $\mathrm{X} 11-5 \mathrm{~A}$ & United States & Oryza sativa cv. Lemont & X. oryzae & C. Gonzales \\
\hline KACC10331 & Korea & O. sativa & X. oryzae pv. oryzae & S. H. Choi \\
\hline BLS413 & Philippines & O. sativa & X. oryzae pv. oryzicola & C. M. Vera Cruz \\
\hline $136 \mathrm{M}$ & Philippines & O. sativa & Unknown, isolated from seed & C. M. Vera Cruz \\
\hline SHU 36 & Philippines & O. sativa & Unknown, isolated from seed & C. M. Vera Cruz \\
\hline SHU 50 & Philippines & O. sativa & Unknown, isolated from seed & C. M. Vera Cruz \\
\hline SHU 100 & Philippines & O. sativa & Unknown, isolated from seed & C. M. Vera Cruz \\
\hline SHU 147 & Philippines & O. sativa & Unknown, isolated from seed & C. M. Vera Cruz \\
\hline SHU 178 & Philippines & O. sativa & Unknown, isolated from seed & C. M. Vera Cruz \\
\hline SHU 202 & Philippines & O. sativa & Unknown, isolated from seed & C. M. Vera Cruz \\
\hline SHU 222 & Philippines & O. sativa & Unknown, isolated from seed & C. M. Vera Cruz \\
\hline SHU 268 & Philippines & $O$. sativa & Unknown, isolated from seed & C. M. Vera Cruz \\
\hline SHU 303 & Philippines & O. sativa & Unknown, isolated from seed & C. M. Vera Cruz \\
\hline M136 & Mali & O. sativa & Xanthomonas sp., isolated from rice & V. Verdier \\
\hline SHU 36 & Philippines & O. sativa & Xanthomonas sp., isolated from seed & C. M. Vera Cruz \\
\hline SHU 50 & Philippines & O. sativa & Xanthomonas sp., isolated from seed & C. M. Vera Cruz \\
\hline SHU 100 & Philippines & $O$. sativa & Xanthomonas sp., isolated from seed & C. M. Vera Cruz \\
\hline SHU 147 & Philippines & O. sativa & Xanthomonas sp., isolated from seed & C. M. Vera Cruz \\
\hline SHU 178 & Philippines & O. sativa & Xanthomonas sp., isolated from seed & C. M. Vera Cruz \\
\hline SHU 202 & Philippines & O. sativa & Xanthomonas sp., isolated from seed & C. M. Vera Cruz \\
\hline SHU 222 & Philippines & O. sativa & Xanthomonas sp., isolated from seed & C. M. Vera Cruz \\
\hline SHU 268 & Philippines & O. sativa & Xanthomonas sp., isolated from seed & C. M. Vera Cruz \\
\hline SHU 303 & Philippines & O. sativa & Xanthomonas sp., isolated from seed & C. M. Vera Cruz \\
\hline MB232 & Cambodia & O. sativa & Pseudomonas sp., isolated from plant & E. Cother \\
\hline MB234 & Cambodia & O. sativa & Pseudomonas sp., isolated from plant & E. Cother \\
\hline MB235 & Cambodia & O. sativa & Pseudomonas sp., isolated from plant & E. Cother \\
\hline MB236 & Cambodia & O. sativa & Pseudomonas sp., isolated from plant & E. Cother \\
\hline MB239 & Cambodia & O. sativa & P. fulva, isolated from plant & E. Cother \\
\hline MB245 & Cambodia & O. sativa & Pseudomonas sp., isolated from plant & E. Cother \\
\hline MB246 & Cambodia & O. sativa & Pseudomonas sp., isolated from plant & E. Cother \\
\hline MB247 & Cambodia & O. sativa & Pseudomonas sp., isolated from plant & E. Cother \\
\hline MB248 & Cambodia & O. sativa & Pseudomonas sp., isolated from plant & E. Cother \\
\hline MB249 & Cambodia & O. sativa & Pseudomonas sp., isolated from plant & E. Cother \\
\hline MB250 & Cambodia & O. sativa & Pseudomonas sp., isolated from plant & E. Cother \\
\hline MB251 & Cambodia & O. sativa & Pseudomonas sp., isolated from plant & E. Cother \\
\hline MB290 & Cambodia & O. sativa & P. plecoglossicida, isolated from plant & E. Cother \\
\hline MB292 & Cambodia & O. sativa & P. tolaasii, isolated from plant & E. Cother \\
\hline DAR 49869 & United States & Quercus sp. & P. putida & Herb DAR \\
\hline DAR 73902 & Australia & Soil & P. putida & Herb DAR \\
\hline DAR 77215 & Australia & Soil & P. putida & Herb DAR \\
\hline
\end{tabular}

\footnotetext{
a All strains were negative with LAMP primer Pf8.
}

b National Collection of Plant Pathogenic Bacteria. 
the phylogenetic tree based on multilocus sequence typing of the eight concatenated gene sequences from $P$. fuscovaginae and other Pseudomonas spp. (Fig. 2). P. fuscovaginae strain SE1 clustered with $P$. stutzeri (with strong bootstrap support) while Pseudomonas sp. (DAR 77817) was distinct, clustering with the P. syringae pv. tomato strain. All P. fuscovaginae strains clustered with strain SE1 from the Philippines based on the concatenated sequences of the entire genomes (Fig. 3). Additionally, strain SE1 was least related to other $P$. fuscovaginae strains. The Pseudomonas sp. strain from Cambodia clustered with P. putida (Fig. 3).

LAMP bioassay. Four highly conserved sequences among $P$. fuscovaginae strains but divergent from other bacterial species were identified after MUMmer alignment of available $P$. fuscovaginae and other bacterial genomes. LAMP primer design software (LAMP Designer; Premier Biosoft) generated five best-match primers for each of the five sequences. Five of the initial F3/B3 primers consistently amplified (primer sets Pf3, Pf4, Pf6, Pf7, and Pf8) a single band of approximately $200 \mathrm{bp}$. Therefore, full sets of LAMP primers were generated based on these sequences. The primer sequences and their relative position in the target are shown in Table 2. No amplification was observed in negative controls that lacked template DNA or contained $P$. syringae DNA (Table 3). Primer set Pf7 failed to amplify DNA from strains DAR 77795 and SE1 and, therefore, was excluded from further analysis. Primer set Pf9 amplified DNA from DAR 77795 and SE1 but failed to amplify DNA from any of the other $P$. fuscovaginae strains. Primer set Pf4 consistently amplified DNA from the Australian $P$. fuscovaginae strains DAR 77795 and DAR 77797 and the Japanese strain ICMP 5940 but gave inconsistent results for strains from the Philippines, Colombia, Madagascar, and Burundi. The primer sets Pf3 and Pf8 amplified most of the tested P. fuscovaginae strains consistently. However, primer set Pf3 failed to amplify Colombian strain UPB 898 from rice while the primer set Pf8 failed to amplify
DNA from the Burundi (UPB 407) strain from the aquatic weed Leersia hexandra.

Primer set Pf8 consistently amplified DNA from strain SE1 when mixed with DNA from a range of Pseudomonas spp. Furthermore, amplification was not detected with this primer set when $P$. fuscovaginae DNA was not included in the pool (negative control). Additionally, amplification was not detected with genomic DNA from other Pseudomonads from rice. When the DNA of $P$. fuscovaginae strain SE1 was serially diluted, the assay detected a concentration of DNA at $1 \mathrm{pg} / \mu \mathrm{l}$ in all samples tested. Using live cells of strain DAR 77795, the assay detected approximately 16 bacterial

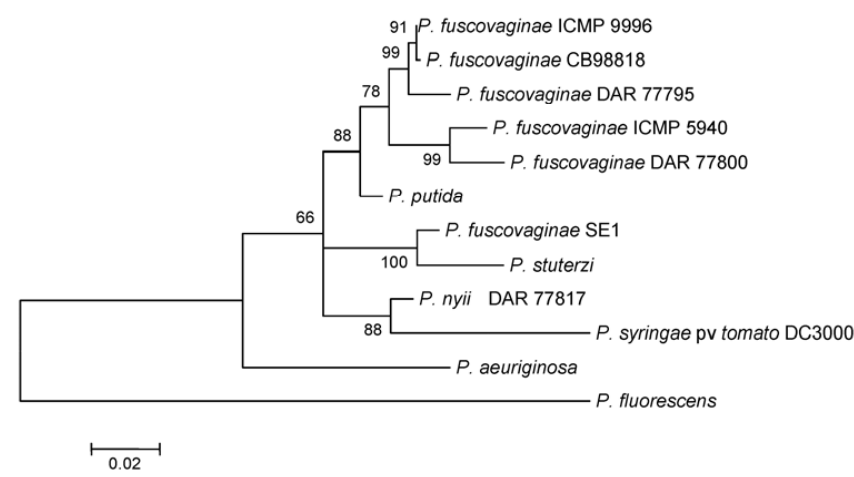

Fig. 2. Molecular phylogenetic analysis of Pseudomonas species by maximumlikelihood (ML) method using the genes acsA (Acetyl coenzyme A synthetase), aroE (Shikimate dehydrogenase), guaA (GMP synthase), mutL (DNA mismatch repair protein), nuoD (NADH dehydrogenase I chain C, D), ppsA (Phosphoenolpyruvate synthase), trpE (Anthralite synthetase component I), and rpoB ( $\beta$ subunit of RNA polymerase). The evolutionary history was inferred by using the ML method. The tree with the highest log likelihood $(-10,674.52)$ is shown.

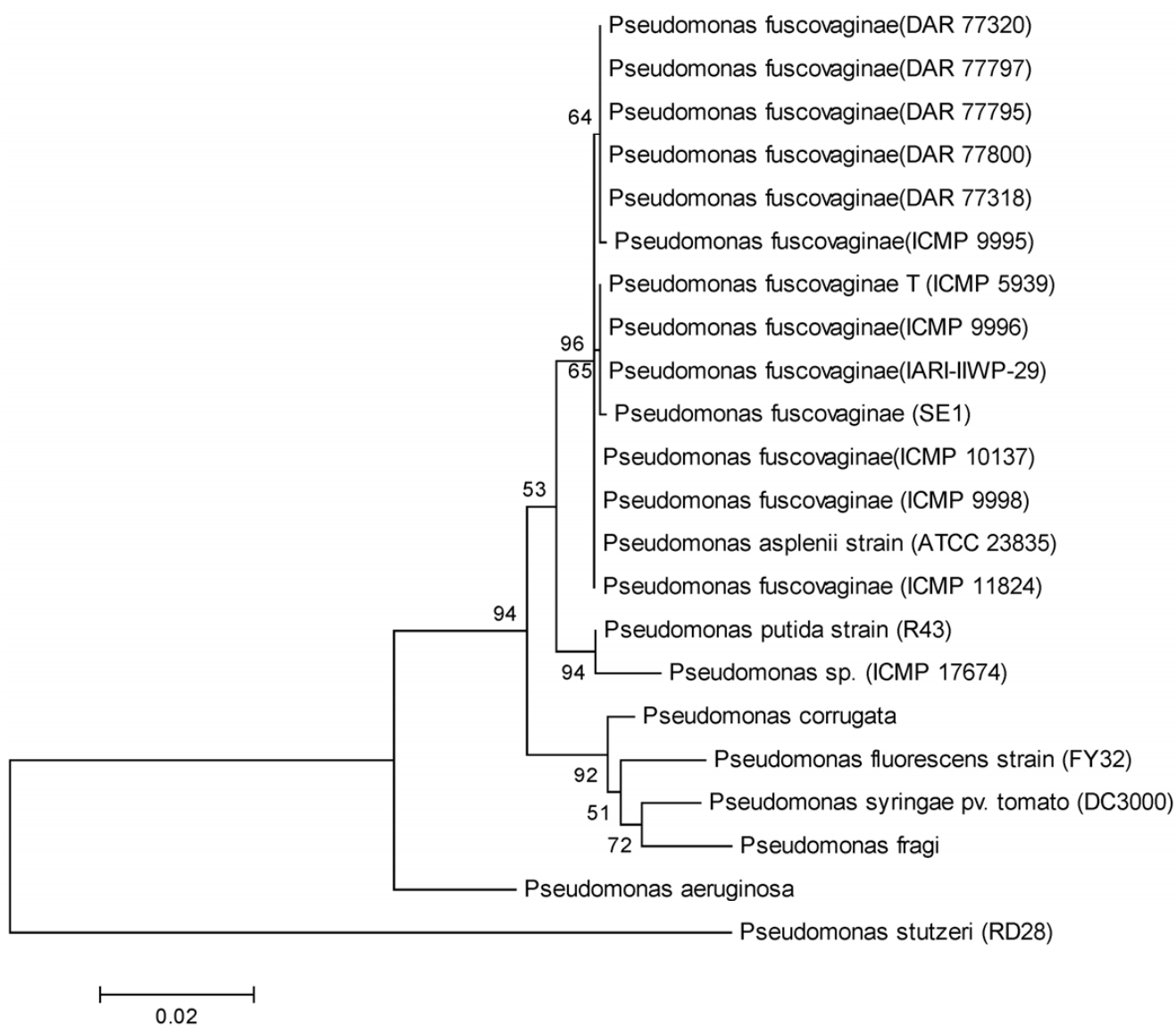

Fig. 1. Molecular phylogenetic analysis of the $16 \mathrm{~s}$ ribosomal RNA gene inferred using the maximum-likelihood method. The tree is drawn to scale, with branch lengths measured in number of substitutions per site. Only bootstrap values (1,000 replicates) greater than $50 \%$ are shown next to branches. 
cells in a $1-\mu \mathrm{l}$ aliquot in all samples tested. The bacterial cell concentration and the number PCR cycles required to reach the minimum threshold were correlated $\left(R^{2}=0.9722\right.$; Fig. 4).

Rice plants inoculated with $P$. fuscovaginae strain DAR 77795 showed typical symptoms of brown sheath rot 5 days after inoculation. Serial dilutions of all the inoculated plant extract showed amplification at all dilutions, whereas there was no amplification from the water-inoculated controls.

\section{Discussion}

The number and diversity of whole-genome sequences of plantpathogenic bacteria continues to increase rapidly, generating huge quantities of data. Many of these genomes are in a draft form and require extensive annotation and comparative analysis. However, draft genomes can be used to find sequences that are unique to species or pathovars of bacterial plant pathogens $(7,24,25)$. Comparative programs such as EDGAR (6) have been used to rapidly identify genomic regions that are unique to a particular species or pathovar which, in turn, can then be used to design LAMP primers (7). The inclusivity of the assay designed using this genomic approach is dependent on the selection of strains that represent the genetic diversity within the group to be analyzed. This is particularly important for Pseudomonads, which are a biologically and genetically diverse group (38).

In this study, we report the draft genome of a strain of $P$. fuscovaginae (SE1) from the Philippines, which was used in conjunction with previously published genomes of other strains of the bacterium $(32,42)$ to develop a robust LAMP assay. This assay was specific to $P$. fuscovaginae-associated sheath brown rot of rice. The $16 \mathrm{~s}$ rRNA gene is traditionally used to ascribe a bacterial strain to a genus (35) but it is incapable of resolving strains below the genus

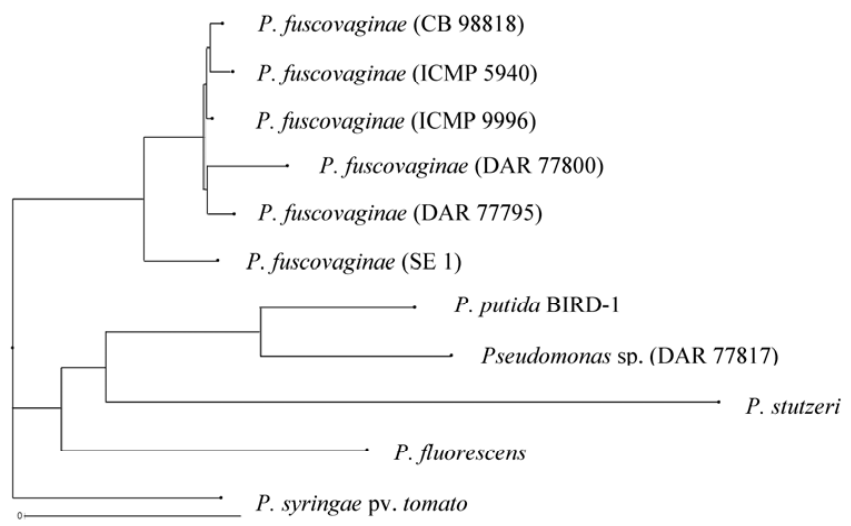

Fig. 3. Phylogenetic tree of Pseudomonas fuscovaginae based on the concatenated sequences of the genomes using the programs EDGAR and DARWin $(5,32)$.

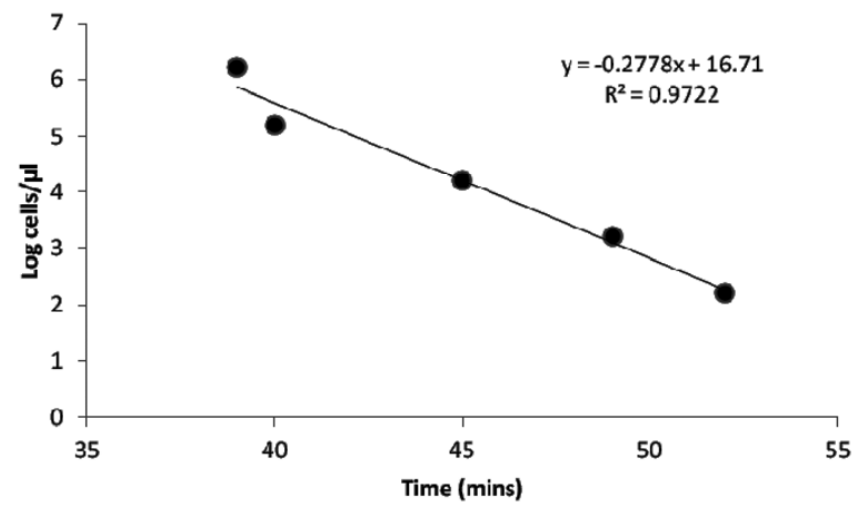

Fig. 4. Correlation between time required to detect Pseudomonas fuscovaginae by loop-mediated isothermal amplification assay and the number of bacterial cells per microliter. level $(4,43)$. The initial phylogenetic analysis of the 16s rRNA gene of Pseudomonas spp. (Fig. 1) showed that the strains chosen for this study clustered with the P. fuscovaginae type strain. This supports the work of Cother et al. (10), who reported the presence of $P$. fuscovaginae in Australia. Strain SE1, first isolated from the Philippines (12) and shown to be highly pathogenic to rice (1), is also a member of this group. However, based on MLST, the SE1 strain is more closely related to P. stutzeri. MLST is often used to increase the resolution of phylogenetic analysis using several housekeeping genes $(4,29,43)$. Finally, concatenated genes from the available draft genomes of $P$. fuscovaginae supported the inclusion of strain SE1 within the species, and helped to define the diversity among the species. This knowledge was used to develop the LAMP assay. Relative to conventional PCR, LAMP is highly specific (due, in part, to the use of six primers), it is an isothermal reaction, and the DNA yields allow visualization using in-tube staining (25). However, the high yield of DNA also presents a problem, because DNA contained in aerosols may yield false-positive results. This can be avoided by the use of systems that do not require the tube to be opened after amplification (such as the Genie II system), or the use of a system where the dye is brought into contact with the DNA after amplification by centrifugation or shaking (18). These approaches will be particularly important when attempting to deploy these assays in the field.

The use of strain SE1 (the most divergent strain for which a draft whole-genome sequence is available) in the initial identification of target genomic DNA contributed to the specificity of the LAMP assay. No false positives were observed when the assay was tested with DNA from over 30 strains of other bacteria often associated with rice and rice seed. Furthermore, the assay differentiated closely related strains that were isolated from hosts other than rice and from the closely related Pseudomonas sp. recently reported from Cambodia (9). The assay detected a concentration of 16 cells of $P$. fuscovaginae per LAMP reaction, which is a similar level of sensitivity reported for the detection of E. amylovora (8). The high sensitivity of LAMP assays is useful when determining the presence of a potential human pathogen in water or clinical samples; however, this may be a drawback for application in the field for routine identification or epidemiological studies of plant pathogens, because there are many opportunities for cross-contamination.

In summary, using comparative genomics of a worldwide collection of strains of $P$. fuscovaginae, we developed LAMP primers that are specific for strains of $P$. fuscovaginae isolated from rice in South America, Asia, Australia, and Africa. The availability of draft whole-genome sequences increases the number of available targets for the development of LAMP diagnostics, which provides opportunity for greater specificity or inclusivity and wider applications for the assays in the future. These primers could be used to confirm isolate identity of bacteria from culture collections and for epidemiological studies and disease surveys, or to assist in the restriction of movement of this important pathogen into new areas of rice production.

\section{Acknowledgments}

We thank Charles Sturt University and the Graham Centre for Agricultural Innovation for funding; and the Colorado State University Agricultural Experiment Station for supporting J. E. Leach, L. R. Triplett, and J. M. Lang.

\section{Literature Cited}

1. Adorada, D. L., Stodart, B. J., Cruz, C. V., Gregorio, G., Pangga, I., and Ash, G. J. 2013. Standardizing resistance screening to Pseudomonas fuscovaginae and evaluation of rice germplasm at seedling and adult plant growth stages. Euphytica 192:1-16.

2. Adorada, D. L., Stodart, B. J., Tpoi, R. P., Costa, S. S., and Ash, G. J. Bacteria associated with sheath browning and grain discoloration of rice in East Timor and implications for Australia's biosecurity. Australas. Plant Dis. Notes 8:43-47.

3. Alonso, A., Rojo, F., and Martinez, J. L. 1999. Environmental and clinical isolates of Pseudomonas aeruginosa show pathogenic and biodegradative properties irrespective of their origin. Environ. Microbiol. 1:421-430.

4. Anzai, Y., Kim, H., Park, J. Y., Wakabayashi, H., and Oyaizu, H. 2000. Phylogenetic affiliation of the pseudomonads based on 16S rRNA sequence. In- 
ternational J. Syst. Evol. Microbiol. 50:1563-1589.

5. Black, R., Holt, J., and Sweetmore, A. 1996. BACTID Bacteriological Identification for Resource-poor Plant Pathology Laboratories: Incorporating BACTID Kit and Expert System. Practical Action Publishing, Chatham, UK.

6. Blom, J., Albaum, S. P., Doppmeier, D., Puehler, A., Vorhoelter, F.-J., Zakrzewski, M., and Goesmann, A. 2009. EDGAR: A software framework for the comparative analysis of prokaryotic genomes. BMC Bioinf. 10:154-169.

7. Bühlmann, A., Pothier, J. F., Rezzonico, F., Smits, T. H. M., Andreou, M., Boonham, N., Duffy, B., and Frey, J. E. 2013. Erwinia amylovora loopmediated isothermal amplification (LAMP) assay for rapid pathogen detection and on-site diagnosis of fire blight. J. Microbiol. Methods 92:332-339.

8. Bühlmann, A., Pothier, J. F., Tomlinson, J. A., Frey, J. E., Boonham, N., Smits, T. H. M., and Duffy, B. 2013. Genomics-informed design of loopmediated isothermal amplification for detection of phytopathogenic Xanthomonas arboricola pv. pruni at the intraspecific level. Plant Pathol. 62:475-484.

9. Cother, E. J., Noble, D. H., van de Ven, R. J., Lanoiselet, V., Ash, G., Vuthy, N., Visarto, P., and Stodart, B. 2010. Bacterial pathogens of rice in the Kingdom of Cambodia and description of a new pathogen causing a serious sheath rot disease. Plant Pathol. 59:944-953.

10. Cother, E. J., Stodart, B., Noble, D. H., Reinke, R., and van de Ven, R. J. 2009. Polyphasic identification of Pseudomonas fuscovaginae causing sheath and glume lesions on rice in Australia. Australas. Plant Pathol. 38:247-261.

11. Cottyn, B., Cerez, M. T., VanOutryve, M. F., Barroga, J., Swings, J., and Mew, T. W. 1996. Bacterial diseases of rice .1. Pathogenic bacteria associated with sheath rot complex and grain discoloration of rice in the Philippines. Plant Dis. 80:429-437.

12. Cottyn, B. J. G. 2003. Bacteria Associated with Rice Seed from Philippine Farmers' Fields. Universiteit Gent, Belgium.

13. Duveiller, E., and Maraite, H. 1990. Bacterial sheath rot of wheat caused by Pseudomonas fuscovaginae in the highlands of Mexico. Plant Dis. 74:932935.

14. Duveiller, E., Snacken, F., Maraite, H., and Autrique, A. 1989. 1st detection of Pseudomonas fuscovaginae on maize and sorghum in Burundi. Plant Dis. 73:514-517.

15. Fan, Q., Xie, Z. X., Xie, L. J., Liu, J. B., Pang, Y. S., Deng, X. W., Xie, Z. Q., Peng, Y., and Wang, X. Q. 2012. A reverse transcription loopmediated isothermal amplification method for rapid detection of bovine viral diarrhea virus. J. Virol. Methods 186:43-48.

16. Hamburger, J., Abbasi, I., Kariuki, C., Wanjala, A., Mzungu, E., Mungai, P., Muchiri, E., and King, C. H. 2013. Evaluation of loop-mediated isothermal amplification suitable for molecular monitoring of schistosome-infected snails in field laboratories. Am. J. Trop. Med. Hyg. 88:344-351.

17. Hollaway, G. J., Bretag, T. W., and Price, T. V. 2007. The epidemiology and management of bacterial blight (Pseudomonas syringae pv. pisi) of field pea (Pisum sativum) in Australia: a review. Aust. J. Agric. Res. 58:10861099.

18. Hong, M., Zha, L., Fu, W. L., Zou, M. J., Li, W. J., and Xu, D. G. 2012. A modified visual loop-mediated isothermal amplification method for diagnosis and differentiation of main pathogens from Mycobacterium tuberculosis complex. World. J. Microbiol. Biotechnol. 28:523-531.

19. Jaunet, T., Laguerre, G., Lemanceau, P., Frutos, R., and Notteghem, J. L. 1995. Diversity of Pseudomonas fuscovaginae and other fluorescent pseudomonads isolated from diseased rice. Phytopathology 85:1534-1541.

20. Jones, D. T., Taylor, W. R., and Thornton, J. M. 1992. The rapid generation of mutation data matrices from protein sequences. Comput. Appl. Biosci. $8: 275-282$

21. Kaneko, H., Kawana, T., Fukushima, E., and Suzutani, T. 2007. Tolerance of loop-mediated isothermal amplification to a culture medium and biological substances. J. Biochem. Biophys. Methods 70:499-501.

22. Kurtz, S., Phillippy, A., Delcher, A. L., Smoot, M., Shumway, M., Antonescu, C., and Salzberg, S. L. 2004. Versatile and open software for comparing large genomes. Genome Biol. 5:1519-1533.

23. Larsen, M. V., Cosentino, S., Rasmussen, S., Friis, C., Hasman, H., Marvig, R. L., Jelsbak, L., Sicheritz-Ponten, T., Ussery, D. W., Aarestrup, F. M., and Lund, O. 2012. Multilocus sequence typing of total-genome-sequenced bacteria. J. Clin. Microbiol. 50:1355-1361.

24. Li, X., Nie, J., Ward, L., Madani, M., Hsiang, T., Zhao, Y., and De Boer, S. H. 2009. Comparative genomics-guided loop-mediated isothermal amplification for characterization of Pseudomonas syringae pv. phaseolicola. J. Appl. Microbiol. 107:717-726.

25. Li, X., Nie, J. B., Ward, L. J., Nickerson, J., and De Boer, S. H. 2011. Development and evaluation of a loop-mediated isothermal amplification assay for rapid detection and identification of Pectobacterium atrosepticum. Can. J. Plant Pathol.-Rev. Can. Phytopathol. 33:447-457.

26. Loper, J. E., Hassan, K. A., Mavrodi, D. V., Davis, E. W., Lim, C. K., Shaf- fer, B. T., Elbourne, L. D. H., Stockwell, V. O., Hartney, S. L., Breakwell, K., Henkels, M. D., Tetu, S. G., Rangel, L. I., Kidarsa, T. A., Wilson, N. L., de Mortel, J. E. V., Song, C. X., Blumhagen, R., Radune, D., Hostetler, J. B., Brinkac, L. M., Durkin, A. S., Kluepfel, D. A., Wechter, W. P., Anderson, A. J., Kim, Y. C., Pierson, L. S., Pierson, E. A., Lindow, S. E., Kobayashi, D. Y., Raaijmakers, J. M., Weller, D. M., Thomashow, L. S., Allen, A. E., and Paulsen, I. T. 2012. Comparative genomics of plant-associated Pseudomonas spp.: insights into diversity and inheritance of traits involved in multitrophic interactions. PLoS Genet. 8.

27. Miyajima, K., Tanii, A., and Akita, T. 1983. Pseudomonas fuscovaginae sp. nov., nom rev Int. J. Syst. Bacteriol. 33:656-657.

28. Mori, Y., Kanda, H., and Notomi, T. 2013. Loop-mediated isothermal amplification (LAMP): recent progress in research and development. J. Infect. Chemother. 19:404-411.

29. Mulet, M., Lalucat, J., and Garcia-Valdes, E. 2010. DNA sequence-based analysis of the Pseudomonas species. Environ. Microbiol. 12:1513-1530.

30. Niessen, L. 2013. Loop-mediated isothermal amplification-based detection of Fusarium graminearum. Methods Mol. Biol. 968:177-193.

31. Notomi, T., Okayama, H., Masubuchi, H., Yonekawa, T., Watanabe, K., Amino, N., and Hase, T. 2000. Loop-mediated isothermal amplification of DNA. Nucleic Acids Res. 28(12)e63.

32. Patel, H. K., da Silva, D. P., Devescovi, G., Maraite, H., Paszkiewicz, K., Studholme, D. J., and Venturi, V. 2012. Draft genome sequence of Pseudomonas fuscovaginae, a broad-host-range pathogen of plants. J. Bacteriol. 194:2765-2766.

33. Perrier, X., and Jacquemoud-Collet, J. P. 2006. DARwin Software. http://darwin.cirad.fr/

34. Rott, P., Notteghem, J. L., and Frossard, P. 1989. Identification and characterization of Pseudomonas fuscovaginae, the causal agent of bacterial sheath brown rot of rice, from Madagascar and other countries. Plant Dis. 73:133-137.

35. Santos, S. R., and Ochman, H. 2004. Identification and phylogenetic sorting of bacterial lineages with universally conserved genes and proteins. Environ. Microbiol. 6:754-759.

36. Sharma, S., Sthapit, B. R., Pradhanang, P. M., and Joshi, K. D. 1997. Bacterial sheath brown rot of rice caused by Pseudomonas fuscovaginae in $\mathrm{Ne}-$ pal. Pages 107-112 in: Conference Rice for Highlands. C. Poisson and J. Rakotoarisoa, eds. Proc. CIRAD Conf Antananarivo, Madagascar.

37. Shi, J. Y., Liu, A. Y., Li, X. P., and Chen, W. X. 2013. Control of Phytophthora nicotianae disease, induction of defense responses and genes expression of papaya fruits treated with Pseudomonas putida MGP1. J. Sci. Food Agric. 93:568-574.

38. Silby, M. W., Winstanley, C., Godfrey, S. A. C., Levy, S. B., and Jackson, R. W. 2011. Pseudomonas genomes: diverse and adaptable. FEMS Microbiol. Rev. 35:652-680.

39. Tamura, K., and Nei, M. 1993. Estimation of the number of nucleotide substitutions in the control region of mitochondrial DNA in humans and chimpanzees. Mol. Biol. Evol. 10:512-526.

40. Tamura, K., Peterson, D., Peterson, N., Stecher, G., Nei, M., and Kumar, S 2011. MEGA5: molecular evolutionary genetics analysis using maximum likelihood, evolutionary distance, and maximum parsimony methods. Mol. Biol. Evol. 28:2731-2739.

41. Tanii, A., Miyajima, K., and Akita, T. 1976. The sheath brown rot disease of rice plant and its causal bacterium, Pseudomonas fuscovaginae A. Tanii, K. Miyajima \& T. Akita sp. nov. Ann. Phytopathol. Soc. Jpn. 42:540-548.

42. Xie, G., Cui, Z., Tao, Z., Qiu, H., Liu, H., Ibrahim, M., Zhu, B., Jin, G., Sun, G., Almoneafy, A., and Li, B. 2012. Genome sequence of the rice pathogen Pseudomonas fuscovaginae CB98818. J. Bacteriol. 194:5479-5480.

43. Yamamoto, S., and Harayama, S. 1998. Phylogenetic relationships of Pseudomonas putida strains deduced from the nucleotide sequences of gyrB, ropD and 16S rRNA genes. Int. J. Syst. Bacteriol. 48:813-819.

44. Yamazaki, W. 2013. Sensitive and rapid detection of Campylobacter jejun and Campylobacter coli using loop-mediated isothermal amplification. Methods Mol. Biol. 943:267-77.

45. Yang, J. L., Zhang, S. H., Liu, Z. H., Yang, R., Huang, Y., and Wen, M. 2012. Development and evaluation of a loop-mediated isothermal amplification assay for the rapid detection of porcine cytomegalovirus under field conditions. Virol. J. 9:321-321.

46. Yasuhara-Bell, J., Kubota, R., Jenkins, D. M., and Alvarez, A. M. 2013. Loop-mediated amplification of the Clavibacter michiganensis subsp. michiganensis micA gene is highly specific. Phytopathology 103:12201226.

47. Zeigler, R. S., and Alvarez, E. 1987. Bacterial sheath brown rot of rice caused by Pseudomonas fuscovaginae in Latin America. Plant Dis. 71:592597.

48. Zeigler, R. S., and Alvarez, E. 1990. Characteristics of Pseudomonas spp. causing grain discoloration and sheath rot of rice, and associated Pseudomonad epiphytes. Plant Dis. 74:917-922. 